\section{Prevalência, conhecimento, tratamento e controle de hipertensão arterial sistêmica na população adulta urbana de Tubarão, Santa Catarina, Brasil, em 2003}

\author{
Prevalence, awareness, treatment, and control \\ of hypertension in the adult urban population of \\ Tubarão, Santa Catarina, Brazil, 2003
}

\author{
${ }_{1}$ Universidade Federal \\ de Santa Catarina, \\ Florianópolis, Brasil. \\ 2 Universidade do Sul de \\ Santa Catarina, Tubarão, \\ Brasil. \\ Correspondência \\ M. R. Pereira \\ Universidade do Sul de \\ Santa Catarina. \\ Rua Otto Fuerchuette 500 \\ apto. 301, Tubarão, SC \\ 88705-020, Brasil. \\ marciarp@tubanet.com.br
}

\begin{abstract}
The aim of this study was to estimate the prevalence, awareness, treatment, and control of systemic arterial hypertension in the adult urban population of Tubarão, Santa Catarina, Brazil. The cross-sectional approach used a stratified sample, based on an initial list of electric power connections, resulting in the selection of 707 individuals over 18 years of age. A questionnaire was applied and arterial blood pressure was measured twice by medical students during home visits, using a mercury sphygmomanometer. The prevalence of arterial hypertension based on the Seventh Report of the Joint National Committee on the Prevention, Detection, Evaluation, and Treatment of High Blood Pressure (arterial pressure $\geq 140 \times 90 \mathrm{mmHg}$ ) was $36.4 \%$, increasing to $40.5 \%$ when including individuals on anti-hypertensive medication. Of the patients with high blood pressure, 55.6\% were aware of their hypertension, $46.8 \%$ were on medication, and $10.1 \%$ had their blood pressure under control. The high estimated prevalence of hypertension, low level of unawareness, and less than ideal treatment and control levels emphasize the need for preventive measures in Tubarão.
\end{abstract}

Hypertension; Prevalence; Adult Health

\author{
Marcia Regina Pereira 1,2 \\ Mário Sérgio Soares de Azeredo Coutinho ${ }^{1,2}$ \\ Paulo F. Freitas 1,2 \\ Eleonora D'Orsi 1 \\ André Bernardi 2 \\ Rafael Hass ${ }^{2}$
}

\section{Introdução}

A hipertensão arterial sistêmica (HAS) é um dos fatores de risco mais importantes para o desenvolvimento de várias doenças cardiovasculares, cerebrovasculares e para a insuficiência renal 1,2. As doenças cardiovasculares constituem um importante problema de Saúde Pública e são as principais causas de morte no Brasil e no mundo ${ }^{3}$.

Dados de saúde internacionais mostram inadequado rastreamento, tratamento e controle dos fatores de risco para doenças cardiovasculares, incluindo a hipertensão arterial, o que contribui substancialmente para a morbidade e a mortalidade por esta doença 4 .

O estabelecimento da prevalência desta condição de risco tem sido uma preocupação em diversos países 5,6,7,8. Existe na literatura uma variação ampla na prevalência de HAS, dependendo da população estudada, do critério adotado para definição de HAS e do método de aferição $9,10,11,12,13,14,15,16,17,18,19,20,21,22,23,24$.

Os estudos existentes sobre a prevalência de HAS no Brasil são limitados a avaliações em algumas cidades e estados e mostram uma prevalência que varia de $10 \%$ a $44 \%$, dependendo da população estudada e do critério adotado para o diagnóstico de HAS 25,26. Poucos desses estudos avaliaram o nível de conhecimento, tratamento e controle da hipertensão 11,12,16,17,22,23. 
Em Tubarão, cidade situada no sul de Santa Catarina, não existem dados sobre prevalência de HAS e, segundo informações do Departamento de Informática do SUS (DATASUS), as doenças cardiovasculares corresponderam a $17,5 \%$ das internações hospitalares e a $30 \%$ da mortalidade geral no ano de 2001, neste município 27.

Portanto, a hipertensão consiste em um grande problema de Saúde Pública e este estudo tem por objetivo descrever a prevalência, conhecimento, tratamento e controle da HAS em Tubarão, de acordo com sexo e faixa etária, visando a contribuir para a comparação entre populações e para o planejamento de ações preventivas neste município.

\section{Métodos}

Este é um estudo transversal de base populacional, cuja população do estudo foi constituída por indivíduos de ambos os sexos, maiores que 18 anos de idade, residentes na área urbana de Tubarão. Segundo dados do Instituto Brasileiro de Geografia e Estatística (IBGE) 28 , a população total de Tubarão era de 89.341 habitantes, sendo a população urbana estimada em 69.910 indivíduos.

Optou-se por realizar uma amostragem estratificada proporcional, na qual os domicílios foram estratificados de acordo com o consumo de energia elétrica em três grupos: baixo consumo - até $160 \mathrm{kWh}$; médio consumo - de 161 a $350 \mathrm{kWh}$; alto consumo - acima de 350kWh. Essa estratificação pretendeu distribuir os indivíduos entrevistados proporcionalmente ao nível sócioeconômico, assumindo uma correlação com o consumo de energia e a renda familiar. Os domicílios foram sorteados de maneira aleatória, com base em uma listagem dos "pontos de luz" (apenas domiciliares) da área urbana de Tubarão, fornecida pela CELESC (Centrais Elétricas de Santa Catarina), em número proporcional ao número de pontos de luz em cada estrato de energia. Para esse procedimento, utilizou-se o programa PEPI versão 4.0 (Computer Programs for Epidemiologists: version 4.0; http://www.sagebrushpress. com/pepi) (Tabela1).

O tamanho da amostra foi calculado em 812 indivíduos, também por meio do programa PEPI, levando-se em consideração uma prevalência de HAS estimada em $20 \%$, intervalo de confiança de $95 \%$ (IC95\%), com uma precisão de 3\% e um adicional de perdas de $20 \%$.

O número de domicílios-alvo foi calculado em 342. Inicialmente, considerou-se a média de moradores por domicílio em Tubarão, que, de acordo com o Censo Demográfico de 1991 do IBGE 29, era de 3,72 habitantes, e o percentual, na população, de pessoas na faixa etária do estudo, que era de 63,9\% (2,37 indivíduos por domicílio). Em seguida, dividiu-se o número de indivíduos necessários para compor a amostra pelo número estimado de pessoas na faixa etária do estudo por domicílio (812/2,37), chegando-se ao número de 342. Adicionando as reservas para possíveis perdas, 547 domicílios foram sorteados de maneira aleatória.

Na Figura 1, está representada a distribuição dos domicílios sorteados que estavam ocupados, os que foram excluídos e os que foram visitados. Dessa forma, foram visitados 366 domicílios (84,3\%), e, em cada um deles, pelo menos um dos moradores maior de 18 anos foi entrevistado.

As perdas de domicílios ocupados não entrevistados, em relação ao total de domicílios entrevistados, foram distribuídas de maneira semelhante entre os estratos (perdas ao acaso) 30 .

\section{Procedimentos para coleta de dados}

\section{- Visita domiciliar}

Dos domicílios selecionados, 366 foram visitados no período de abril de 2003 a setembro de 2003, por estudantes de medicina do terceiro ano, pre-

Tabela 1

Distribuição dos domicílios em número total e em número de sorteados, de acordo com a faixa de consumo de energia.

\begin{tabular}{lcccc}
\hline Estrato & $\begin{array}{c}\text { Consumo de } \\
\text { energia }(\mathrm{kWh})\end{array}$ & $\begin{array}{c}\text { Número total } \\
\text { de domicílios }\end{array}$ & $\begin{array}{c}\text { Número de } \\
\text { domicílios sorteados }\end{array}$ \\
\hline I & $\leq 160$ & 9.078 & 240 & 43,73 \\
II & $\geq 161-350$ & 9.403 & 246 & 45,30 \\
III & $>350$ & 2.279 & 61 & 10,97 \\
Total & - & 20.760 & 547 & 100,00 \\
\hline
\end{tabular}


viamente treinados, acompanhados ou orientados por agentes de saúde da região. Todos os domicílios sorteados foram visitados até três vezes, com o objetivo de contatar todos os moradores maiores de 18 anos; algumas das visitas foram realizadas no final de semana.

A finalidade dessas visitas foi realizar uma entrevista para preenchimento de um questionário, no qual foram coletados dados de variáveis biológicas, sociais, e para avaliação do conhecimento prévio de HAS, o tratamento e controle, assim como aferição da pressão arterial, após o consentimento do entrevistado por escrito para participar do estudo.

Para o controle de qualidade dos dados obtidos, a pesquisadora realizou nova visita para conferência dos dados em vinte domicílios aleatoriamente escolhidos, onde foram reintrevistados 39 indivíduos, não havendo diferenças significativas entre os dados coletados pela pesquisadora e seus colaboradores, confirmando a aplicação adequada do questionário.

\section{- Aferição da pressão arterial}

Os entrevistados foram submetidos à medida da pressão arterial de forma padronizada; os critérios adotados para aferição estavam de acordo com os do JNC 6 (The Sixth Report of The Joint National Committee on the Prevention, Detection, Evaluation, and Treatment of High Blood Pressure) ${ }^{31}$.

Foram realizadas duas medidas da pressão arterial: a primeira, cinco minutos após a entrevista, e a segunda, cinco minutos após a primeira medida, estando o indivíduo sentado, no membro superior esquerdo. Caso a diferença entre as duas primeiras medidas fosse maior que $6 \mathrm{mmHg}$, realizavam-se novas medidas até que a diferença fosse inferior a esse valor, e então consideravamse as duas últimas medidas com os valores mais próximos.

A pressão arterial foi medida por manômetros de mercúrio Premium, da GLICOMED, modelo CE 0197, (GLICOMED Produtos Médicos e Hospitalares, Brasil), com precisão de $2 \mathrm{mmHg}$. O manguito foi selecionado após a medida da circunferência braquial, sendo utilizados os tamanhos adulto pequeno, adulto normal e adulto grande, de acordo com tabela publicada pelas IV Diretrizes Brasileiras de Hipertensão Arterial 32, de forma que a largura da bolsa de borracha correspondesse a $40 \%$ da circunferência do braço e o seu comprimento envolvesse pelo menos $80 \%$.
Figura 1

Distribuição dos domicílios selecionados.

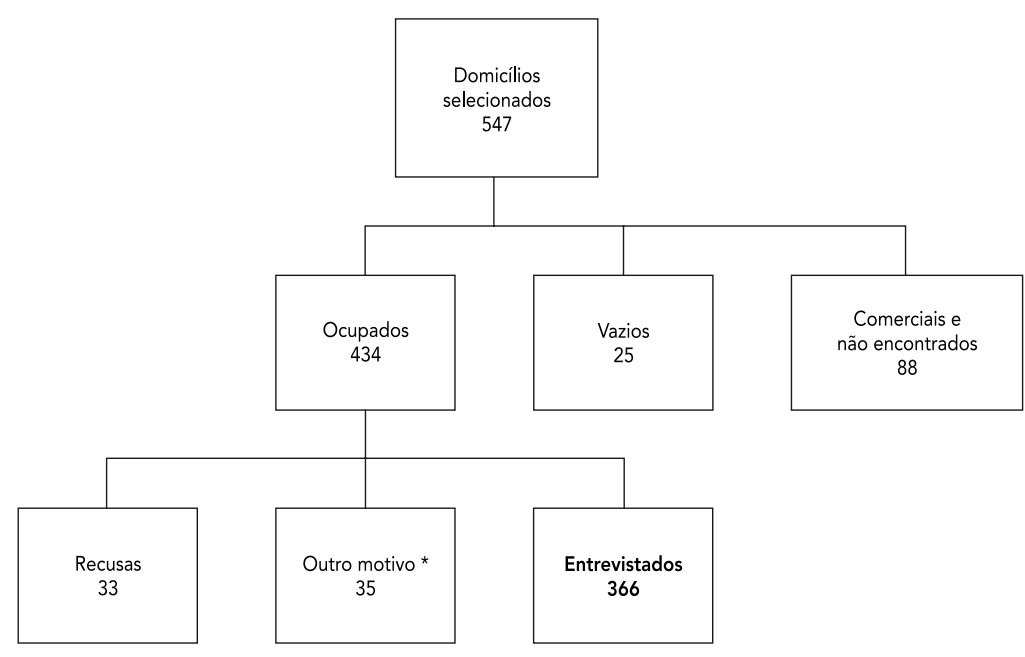

* Outro motivo: 32 não entrevistados após três visitas; três incapazes.

\section{Critérios utilizados}

Foram definidos como hipertensos os indivíduos com a média das duas aferições maior ou igual a 140mmHg para a pressão sistólica e/ou maior ou igual a 90mmHg para pressão diastólica, ou abaixo desses níveis, se estivessem sob uso de algum anti-hipertensivo.

Também de acordo com a classificação do JNC 7 (The Seventh Report of The Joint National Committee on the Prevention, Detection, Evaluation, and Treatment of High Blood Pressure) 3 , os indivíduos da amostra foram distribuídos em: (a) normal - pressão arterial sistólica (PAS) menor que $120 \mathrm{mmHg}$ e pressão arterial diastólica (PAD) menor que 80mmHg; (b) pré-hipertensão - PAS 120-139mmHg e/ou PAD 80-89mmHg; (c) hipertensão estágio 1 (HAS1) - PAS 140159mmHg e/ou PAD 90-99mmHg; (d) hipertensão estágio 2 (HAS2) - PAS $\geq 160 \mathrm{mmHg}$ e/ ou PAD $\geq 100 \mathrm{mmHg}$.

O conhecimento da condição de hipertenso foi caracterizado a partir do diagnóstico prévio da doença por um profissional de saúde entre a população definida como hipertensa 5 .

Para avaliação do tratamento da hipertensão, foi considerado apenas o tratamento farmacológico naqueles indivíduos que se diziam hipertensos. 
O nível de controle da HAS na população foi calculado considerando-se os que se diziam hipertensos, tratavam-se e estavam com a pressão arterial controlada e em relação ao número total de hipertensos.

\section{Análise estatística}

Os dados foram digitados utilizando-se o programa Excel (Microsoft Corp., Estados Unidos) e analisados utilizando-se os programas Epi Info, versão 6.04 (Centers for Disease Control and Prevention, Atlanta, Estados Unidos) e SPSS versão 10.0 (SPSS Inc., Chicago, Estados Unidos). Foram calculados IC95\% para as prevalências estudadas.

A existência de uma associação do tipo "dose-resposta”, ao comparar a relação entre os diferentes níveis de consumo de energia e a renda familiar, foi avaliada utilizando-se o teste do $\chi^{2}$ para um nível de confiança de 95\% ( $<<0,05) 33$.

Foi utilizado o teste $t$ student para avaliar diferenças entre as médias de algumas variáveis estudadas.

Considerando que a proporção de indivíduos em cada faixa etária encontrada na amostra foi diferente da distribuição na população geral, segundo o Censo Demográfico 28 do IBGE, foi realizado o cálculo da prevalência padronizada por idade pelo método direto (prevalência ajustada pela idade). A prevalência padronizada por idade foi obtida aplicando-se a taxa específica de prevalência em cada faixa etária (p1 a p5) no presente estudo para a população geral estimada em cada faixa etária (n1 a n5), segundo o IBGE no ano de 2003 34, dividindo-se pelo total da população estimada (PT) 35 . O cálculo da taxa de prevalência ajustada pela idade foi realizado conforme a fórmula:

$$
\frac{(n 1 x p 1)+(n 5 x p 5)}{P T}
$$

Com base na comparação com a classificação do JNC 73 (considerada padrão ouro), foram calculados os valores de sensibilidade, especificidade, valor preditivo positivo (VPP) e negativo (VPN) e respectivos IC95\% para a pergunta: “ $\mathrm{Al}$ guma vez algum médico disse que o(a) senhor(a) tem pressão alta?", realizada na entrevista.

O projeto de pesquisa e o Consentimento Informado e Esclarecido foram aprovados pelo Comitê de Ética em Pesquisa em Seres Humanos, da Universidade Federal de Santa Catarina, em 2002.

\section{Resultados}

\section{Caracterização da população do estudo}

A amostra populacional foi constituída por 707 indivíduos entrevistados em seus domicílios (Tabela 2). A distribuição da amostra de entrevistados segundo estratos de consumo de energia elétrica foi semelhante à distribuição na população de origem. Houve uma associação significativa entre o consumo de energia per capita e a renda familiar $\left(\chi^{2} ; \mathrm{p}<0,05\right)$.

A distribuição etária da amostra foi equilibrada, com aproximadamente um quinto em cada faixa etária. Houve predomínio significativo, entre os entrevistados, de indivíduos do sexo feminino, cor da pele branca e estado civil casado. As categorias de ocupação predominantes foram trabalho remunerado e aposentado. Em relação ao nível de instrução, 52,8\% tinham ensino fundamental completo ou incompleto. O percentual nos extremos de renda foi pequeno, estando a maioria da população na faixa de 1,1 a 5 salários mínimos (Tabela 2).

A composição etária mais idosa e maior participação feminina na amostra, quando comparada à população de origem, justificam os ajustes realizados, nas prevalências, para essas duas variáveis.

A média de idade foi 45,3 anos (IC95\%: 44,146,3), sendo 45,1 (IC95\%: 43,2-47,0) nos homens e 45,5 (IC95\%: 43,9-47,0) nas mulheres, diferença não significativa $(p=0,78)$. As médias das PAS e PAD foram respectivamente de $129,4 \mathrm{mmHg}$ (IC95\%: 127,8-131,0) e 82,0mmHg (IC95\%: 81,1-82,9). Ambas foram maiores nos homens $(\mathrm{PAS}=131,1 \mathrm{mmHg}$, IC95\%: 128,7-133,5 e PAD $=83,7 \mathrm{mmHg}$, IC95\%: 82,3-85,1) do que nas mulheres (PAS = 128,1mmHg, IC95\%: 126,0-130,3 e $\mathrm{PAD}=80,8 \mathrm{mmHg}$, IC95\%: 79,6-81,9), diferença estatisticamente limítrofe para a PAS $(p=0,07)$ e significativa para PAD $(\mathrm{p}<0,05)$.

\section{Prevalência de HAS segundo a classificação do JNC 7}

A distribuição dos indivíduos segundo os critérios de classificação de HAS do JNC 7 3, de acordo com o sexo e faixa etária (utilizando apenas as medidas da pressão arterial para classificação), está demonstrada na Tabela 3. As prevalências totais são apresentadas de duas maneiras: as obtidas na amostra (brutas) e as estimativas populacionais por sexo (corrigidas para a estrutura etária real da população de cada sexo, separadamente). A prevalência de pré-hipertensão é maior nos indivíduos mais jovens do que nos idosos 
Distribuição dos entrevistados na população urbana de Tubarão, Santa Catarina, Brasil, no ano de 2003, segundo as características sócio-demográficas ( $\mathrm{n}=707$ ).

\begin{tabular}{|c|c|c|c|}
\hline Características sócio-demográficas & $\mathbf{n}$ & $\%$ & IC95\% \\
\hline \multicolumn{4}{|l|}{ Consumo de energia no domicílio (kWh) } \\
\hline$\leq 160$ (estrato I) & 280 & 39,6 & $35,9-43,3$ \\
\hline$\geq 161-350$ (estrato II) & 345 & 48,8 & $45,0-52,5$ \\
\hline$>350$ (estrato III) & 82 & 11,6 & $9,3-14,2$ \\
\hline \multicolumn{4}{|l|}{ Faixa etária (anos) } \\
\hline $18-29$ & 131 & 18,5 & $15,7-21,6$ \\
\hline $30-39$ & 144 & 20,4 & $17,5-23,5$ \\
\hline $40-49$ & 155 & 21,9 & $18,9-25,1$ \\
\hline $50-59$ & 135 & 19,1 & $16,3-22,2$ \\
\hline$\geq 60$ & 142 & 20,1 & $17,2-23,2$ \\
\hline \multicolumn{4}{|l|}{ Sexo } \\
\hline Masculino & 305 & 43,1 & $39,4-46,9$ \\
\hline Feminino & 402 & 56,9 & $53,1-60,5$ \\
\hline \multicolumn{4}{|l|}{ Cor } \\
\hline Branco & 679 & 96,0 & $94,3-97,3$ \\
\hline Não branco & 28 & 3,9 & $2,6-5,7$ \\
\hline \multicolumn{4}{|l|}{ Estado civil } \\
\hline Casado & 491 & 69,4 & $65,9-72,8$ \\
\hline Solteiro & 120 & 17,0 & $14,3-19,9$ \\
\hline Separado/Divorciado & 37 & 5,2 & $3,7-7,1$ \\
\hline Viúvo & 59 & 8,3 & $6,4-10,6$ \\
\hline \multicolumn{4}{|l|}{ Ocupação } \\
\hline Trabalho remunerado & 291 & 41,2 & $37,5-44,9$ \\
\hline Desempregado & 18 & 2,5 & $1,5-3,9$ \\
\hline Do lar & 125 & 17,7 & $14,9-20,7$ \\
\hline Aposentado & 156 & 22,1 & $19,0-25,3$ \\
\hline Estudante & 29 & 4,1 & $2,7-5,8$ \\
\hline Trabalho informal & 64 & 9,1 & $7,0-11,4$ \\
\hline Outro & 24 & 3,4 & $2,2-4,9$ \\
\hline \multicolumn{4}{|l|}{ Nível de instrução } \\
\hline Analfabeto e fundamental incompleto & 257 & 36,4 & $32,8-40,0$ \\
\hline Fundamental completo & 116 & 16,4 & $13,7-19,3$ \\
\hline Ensino médio & 198 & 28,0 & $24,7-31,5$ \\
\hline Superior e pós-graduação & 136 & 19,2 & $16,4-22,3$ \\
\hline \multicolumn{4}{|l|}{ Renda familiar (salários mínimos) } \\
\hline$\leq 1$ & 26 & 3,7 & $2,4-5,3$ \\
\hline $1,1-5,0$ & 359 & 50,8 & $47,0-54,5$ \\
\hline $5,1-10,0$ & 197 & 27,9 & $24,6-31,3$ \\
\hline $10,1-20$ & 85 & 12,0 & $9,8-14,6$ \\
\hline$>20$ & 36 & 5,1 & $3,6-6,9$ \\
\hline Inexistência de dados & 4 & 0,6 & $0,1-1,4$ \\
\hline
\end{tabular}

( $\geq 60$ anos), enquanto a prevalência de hipertensão aumenta com a idade em ambos os sexos. As prevalências ajustadas, tanto de préhipertensão como de hipertensão, foram significativamente maiores nos homens do que nas mulheres.

\section{Conhecimento, tratamento e controle}

Dos 707 indivíduos estudados, 177 se diziam hipertensos. Entre os que se diziam hipertensos, 18 $(10,2 \%)$ tinham a pressão arterial normal e não faziam tratamento; nesse caso, foram considera- 
Distribuição dos níveis de pressão arterial pela classificação do JNC 7 (The Seventh Report of The Joint National Committee on the Prevention, Detection, Evaluation, and Treatment of High Blood Pressure) 3, segundo faixa etária e sexo. Tubarão, Santa Catarina, Brasil, 2003.

\begin{tabular}{|c|c|c|c|c|c|c|c|}
\hline \multirow[t]{3}{*}{ Sexo e faixa etária } & \multirow{3}{*}{$\mathbf{n}$} & \multicolumn{6}{|c|}{ Classificação do JNC 7} \\
\hline & & \multicolumn{2}{|c|}{ Normal * } & \multicolumn{2}{|c|}{ Pré-hipertensão ** } & \multicolumn{2}{|c|}{ Hipertensão *** } \\
\hline & & $\%$ & IC95\% & $\%$ & IC95\% & $\%$ & IC95\% \\
\hline \multicolumn{8}{|l|}{ Masculino } \\
\hline \multicolumn{8}{|l|}{ Faixa etária (anos) } \\
\hline $18-29$ & 58 & 50,0 & $36,3-63,7$ & 36,2 & $22,9-49,4$ & 13,8 & $4,1-23,5$ \\
\hline $30-39$ & 66 & 19,7 & $9,3-30,0$ & 56,1 & $43,3-68,8$ & 24,2 & $13,1-35,3$ \\
\hline $40-49$ & 63 & 14,2 & $4,8-23,7$ & 42,9 & $29,8-55,8$ & 42,9 & $29,8-55,8$ \\
\hline $50-59$ & 56 & 12,5 & $2,9-22,0$ & 35,7 & $22,3-49,1$ & 51,8 & $37,8-65,7$ \\
\hline$\geq 60$ & 62 & 4,8 & $1,0-13,5$ & 27,4 & $15,5-39,3$ & 67,8 & $55,3-80,2$ \\
\hline Total da amostra & 305 & 20,0 & $15,3-24,6$ & 40,0 & $34,3-45,7$ & 40,0 & $34,3-45,7$ \\
\hline Total da população \# & & 25,2 & $24,7-25,7$ & 41,5 & $40,9-42,0$ & 33,3 & $32,7-33,8$ \\
\hline \multicolumn{8}{|l|}{ Feminino } \\
\hline \multicolumn{8}{|l|}{ Faixa etária (anos) } \\
\hline $18-29$ & 73 & 57,5 & $45,5-69,6$ & 37,0 & $25,3-48,7$ & 5,5 & $1,5-13,4$ \\
\hline $30-39$ & 78 & 42,3 & $30,7-53,9$ & 35,9 & $24,6-47,2$ & 21,8 & $11,9-31,6$ \\
\hline $40-49$ & 92 & 35,9 & $25,5-46,2$ & 35,9 & $25,5-46,2$ & 28,2 & $18,5-38,0$ \\
\hline $50-59$ & 79 & 16,5 & $7,6-25,3$ & 39,2 & $27,8-50,6$ & 44,3 & $32,7-55,9$ \\
\hline$\geq 60$ & 80 & 7,5 & $1,1-13,9$ & 26,3 & $15,9-36,5$ & 66,2 & $55,2-77,0$ \\
\hline Total da amostra & 402 & 31,6 & $26,9-36,3$ & 34,8 & $30,0-39,6$ & 33,6 & $28,8-38,3$ \\
\hline Total da população \# & & 37,1 & $36,6-37,6$ & 35,3 & $34,7-35,8$ & 27,6 & $27,1-28,1$ \\
\hline Total geral da amostra & 707 & 26,6 & $23,3-29,9$ & 37,1 & $33,4-40,7$ & 36,4 & $32,7-39,9$ \\
\hline Total geral da população \# & & 32,0 & $31,7-32,4$ & 38,0 & $37,6-38,4$ & 29,9 & $29,6-30,3$ \\
\hline
\end{tabular}

* Normal: pressão arterial sistólica (PAS) $<120 \mathrm{mmHg}$ e pressão arterial diastólica (PAD) $<80 \mathrm{mmHg}$;

** Pré-hipertensão: PAS 120-139mmHg e/ou PAD 80-89mmHg;

*** Hipertensão: PAS $\geq 140 \mathrm{mmHg}$ e/ou PAD $\geq 90 \mathrm{mmHg}$;

\# Prevalências corrigidas para a população indicada pelo Censo Demográfico 46, de 2003, Instituto Brasileiro de Geografia e Estatística (IBGE), segundo sexo.

dos "falsos hipertensos", sendo excluídos da população de hipertensos.

A sensibilidade da pergunta "Alguma vez algum médico disse que o(a) senhor(a) tem pressão alta?" realizada na entrevista, quando comparada à classificação do JNC 73 (considerada padrão ouro), foi igual a 55,6\% (IC95\%: 49,6-61,4) e a especificidade foi de 95,7\% (IC95\%: 93,2-97,4). O VPP foi de 89,8\% (IC95\%: 84,2-93,7) e o VPN foi igual a 76\% (IC95\%: 72,1-79,6). Chama atenção a baixa sensibilidade, com elevado percentual (44,4\%) de indivíduos falso-negativos, ou seja, que desconheciam sua condição de hipertensos. A especificidade foi bem mais elevada, havendo apenas 4,3\% de indivíduos classificados como falso-positivos (que se disseram hipertensos mas estavam com os níveis tensionais normais e não tomavam medicação anti-hipertensiva).

Acrescentando-se os 29 indivíduos que faziam tratamento e estavam com a pressão arte- rial controlada à prevalência de HAS pelo critério de pressão arterial $\geq 140 \times 90 \mathrm{mmHg}(\mathrm{n}=257)$, obteve-se 286 hipertensos (40,5\%).

Dentre os 286 hipertensos, 134 (46,8\%) estavam em tratamento, dos quais $29(10,1 \%)$ estavam controlados e 105 não estavam controlados; 25 não se tratavam e não estavam controlados e $127(44,4 \%)$ não sabiam que eram hipertensos. As mulheres mostraram maior conhecimento a respeito de serem portadoras de HAS, em relação aos homens, apresentavam maior índice de tratamento e estavam mais controladas, porém, neste último item, sem significância estatística (Tabela 4).

\section{Prevalência de hipertensão segundo vários critérios}

Levando-se em consideração apenas pressão arterial $\geq 140 \times$ x 90mmHg, obteve-se $36,4 \%$ (IC95\%: 
Distribuição, por sexo, dos indivíduos hipertensos segundo conhecimento, tratamento e controle da hipertensão. Tubarão, Santa Catarina, Brasil, 2003.

\begin{tabular}{|c|c|c|c|c|c|c|c|c|c|c|c|c|c|}
\hline \multirow[t]{2}{*}{ Sexo } & \multirow{2}{*}{$\begin{array}{c}\text { Total } \\
n\end{array}$} & \multicolumn{3}{|c|}{ Hipertensos * } & \multicolumn{3}{|c|}{ Conhecimento ** } & \multicolumn{3}{|c|}{ 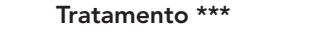 } & \multicolumn{3}{|c|}{ Controle \# } \\
\hline & & $\mathrm{n}$ & $\% \# \#$ & IC95\% & $\mathrm{n}$ & $\%$ \#\# & IC95\% & $\mathrm{n}$ & $\% \S$ & IC95\% & $\mathrm{n}$ & $\% \S \S$ & IC95\% \\
\hline Masculino & 305 & 132 & 43,3 & $37,7-49,0$ & 63 & 47,7 & $39,0-56,5$ & 50 & 37,8 & $29,6-46,7$ & 10 & 20,0 & $10,0-33,7$ \\
\hline Feminino & 402 & 154 & 38,3 & $33,5-43,2$ & 96 & 62,3 & $54,1-69,9$ & 84 & 54,5 & $46,3-62,6$ & 19 & 22,6 & $14,2-33,0$ \\
\hline Total & 707 & 286 & 40,5 & $36,8-44,2$ & 159 & 55,6 & $49,6-61,4$ & 134 & 46,8 & $40,9-52,8$ & 29 & 21,6 & $14,9-29,6$ \\
\hline
\end{tabular}

* Pressão arterial sistólica (PAS) $\geq 140 \mathrm{mmHg}$ e/ou pressão arterial diastólica (PAD) $\geq 90 \mathrm{mmHg}$ mais níveis pressóricos inferiores a 140/90mmHg de indivíduos em tratamento medicamentoso;

** Conhecimento em relação ao diagnóstico de hipertensão;

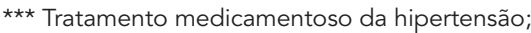

\# Níveis pressóricos inferiores a 140/90mmHg entre aqueles em tratamento;

\#\# Percentual de hipertensos entre os indivíduos entrevistados;

\#\#\# Percentual de conhecimento sobre o diagnóstico entre os indivíduos hipertensos;

$\S$ Percentual de tratamento medicamentoso entre indivíduos hipertensos;

$\S \S$ Percentual de indivíduos com níveis pressóricos inferiores a 140/90mmHg entre aqueles em tratamento medicamentoso para hipertensão.

32,7-39,9) de prevalência de hipertensão na população adulta de Tubarão; quando se levaram em consideração os indivíduos em tratamento controlados, esta foi de 40,5\% (IC95\%: 36,8-44,2). Entre a população hipertensa $(n=286)$, a prevalência de hipertensão arterial sistólica isolada (HASI) foi de 23,8\% (IC95\%: 18,9-29,1); de hipertensão arterial diastólica isolada (HADI), de 20,6\% (IC95\%: 16,0-25,8); de hipertensão sisto-diastólica, de 45,5\% (IC95\%: 39,6-51,4); e de hipertensão controlada, 10,1\% (IC95\%: 6,9-14,2).

A prevalência de HAS ajustada para a idade, pelo critério do JNC 73 , foi de 29,9\% (IC95\%: 29,6$30,3)$.

\section{Discussão}

São numerosos os estudos que estimam a prevalência de HAS no mundo, mas poucos no Brasil. Taxas discrepantes de prevalência nesses estudos podem ser justificadas pelas diferentes metodologias aplicadas - como definições diferentes de HAS (pontos de corte diferentes para definir HAS, inclusão ou não de indivíduos com pressão arterial normal em uso de anti-hipertensivos), variações na população-alvo (faixas etárias diferentes, amostras com grupos selecionados), número de visitas para aferir a pressão -, bem como pelas variações raciais, culturais e sócio-econômicas das populações nas diversas regiões estudadas $25,36,37$.

Birkett 38 avaliou o impacto de vários critérios adotados para definir HAS em estudos populacionais, com o objetivo de estimar a sua prevalência, tratamento e controle, demons trando que variações no número de visitas para medir a pressão arterial e o ponto de corte para definir HAS eram os critérios que tinham maior impacto na estimativa da prevalência e controle da hipertensão. Dessa forma, as prevalências obtidas neste estudo foram comparadas principalmente com as daqueles estudos que utilizaram o mesmo ponto de corte para definição de HAS e em faixa etária semelhante 11,12,14,15,16,17,19,21,23,39.

Esta pesquisa utilizou dados de consumo de energia para compor a amostra em estratos com o objetivo de garantir uma representatividade sócio-econômica da população de Tubarão. Como houve uma associação entre consumo de energia per capita e renda familiar, acredita-se que este objetivo foi alcançado.

\section{Prevalência de HAS segundo a classificação do JNC 7}

Nos Estados Unidos, no período de 1999 a 2000, de acordo com o estudo Third National Health and Nutrition Examination Survey (NHANES III), 29\% dos americanos eram hipertensos e $31 \%$ pré-hipertensos 6 .

De acordo com essa classificação, 37,1\% dos indivíduos avaliados no presente trabalho eram pré-hipertensos, dado que aponta para um percentual elevado de indivíduos em risco de desenvolver HAS e doenças cardiovasculares na população de Tubarão. Assim, a comunidade médica e as instituições que trabalham com a promoção da saúde deverão ser alertadas para que, no que se 
refere aos indivíduos pré-hipertensos, já devam ser tomadas medidas que estimulem mudanças no estilo de vida a fim de obter níveis tensionais mais baixos, diminuindo, com isso, o incremento de doenças cardiovasculares.

A idade constitui um dos principais fatores de risco para hipertensão arterial, com prevalência crescente com o avançar da idade, resultado encontrado neste e em outros estudos nacionais $10,12,15,20,21,23,24,39$.

A prevalência de pré-hipertensão encontrada no NHANES III (1999-2000) foi maior nos homens (40\%) do que nas mulheres (23\%) 6. Em Tubarão, a prevalência de pré-hipertensos no sexo masculino foi semelhante à dos americanos (40\%) e superior no grupo feminino $(34,8 \%)$. Com relação à distribuição em faixas etárias, o resultado foi semelhante ao encontrado no NHANES III, ou seja, uma taxa menor de pré-hipertensos nas idades mais avançadas.

\section{Prevalência de HAS, considerando-se os indivíduos tratados}

Entre os estudos sobre prevalência de HAS no Brasil, selecionaram-se, para análise comparativa, alguns que tiveram como definição de hipertensão pressão arterial $\geq 140$ x 90mmHg 10,11,12,14, 15,16,19,21,23,39. Alguns desses estudos incluíram na definição de hipertensão os indivíduos que estavam em uso de anti-hipertensivo, critério utilizado na maioria dos estudos internacionais 12,14,16,21,23,39. A Tabela 5 mostra as prevalências e as características de tais estudos.

O presente estudo mostra uma elevada prevalência de HAS em Tubarão por ambos os critérios adotados, sendo de 40,5\% quando incluídos os hipertensos em tratamento controlados, com predominância no sexo masculino $(43,3 \%$ no sexo masculino e $38,3 \%$ no sexo feminino).

Em poucos estudos de prevalência realizados no Brasil a amostra foi estratificada levando-se em consideração o nível sócio-econômico da população; nestes, as prevalências pontuais de hipertensão foram maiores 14,16,20,23,39. Como a prevalência de HAS tende a ser maior nas classes mais baixas 20,40 , esse fato pode justificar uma maior prevalência encontrada em Tubarão e nos estudos com definição semelhante de HAS 14,16,23. Segundo um estudo realizado em Vitória, Estado do Espírito Santo, Brasil, a ingestão de sal é altamente influenciada pelo nível sócio-econômico e pode explicar parcialmente a alta prevalência de HAS nas classes sócio-econômicas mais baixas 41 .

Outro determinante para a alta prevalência de HAS em Tubarão pode ser o fato de que a amostra estudada apresentou um percentual maior de idosos (maiores que 60 anos) em relação à população geral do município. Considerando esse fa-

Estudos nacionais de prevalência de hipertensão arterial sistêmica (HAS) (pressão arterial $\geq 140 \times 90 \mathrm{mmHg}$ ) comparados com o estudo realizado em Tubarão, Santa Catarina, Brasil, em 2003.

\begin{tabular}{|c|c|c|c|c|c|c|c|}
\hline Local & Autores & Ano & Amostra & Idade & $\begin{array}{l}\text { Medidas/ } \\
\text { Visitas }\end{array}$ & Aparelho & $\begin{array}{c}\text { Prevalência } \\
\text { (\%) }\end{array}$ \\
\hline Porto Alegre & Fuchs et al. 12 & 1995 & 1.091 & $\geq 18$ & $2 / 1$ & Aneróide \# & 29,8 ** \\
\hline Campos dos Goytacazes & Souza et al. 16 & 2001 & 1.039 * & $\geq 18$ & $2 / 2$ & Aneróide & $30,5 * \star$ \\
\hline Catanduva & Freitas et al. 11 & 2001 & 688 & $\geq 18$ & $2 / 1$ & Aneróide & 31,5 \\
\hline Rio Grande do Sul & Gus et al. 17 & 2002 & $1.066 * \star \star$ & $\geq 20$ & $2 / 1$ & $\mathrm{NI}$ & 31,6 \\
\hline Piracicaba & Ayres 15 & 1991 & 1.900 & $\geq 15$ & $\mathrm{NI} / 1$ & $\mathrm{NI}$ & 32,7 \\
\hline Tubarão & - & 2003 & 707 * & $\geq 18$ & $2 / 1$ & Mercúrio & $40,5 * \star$ \\
\hline Cotia & Salas Martins et al. 14 & 1990, 1991 & $1.041 *$ & $20-88$ & $\mathrm{NI}$ & Mercúrio & 44,4 ** \\
\hline São Paulo & Marcopito et al. 19 & 2001, 2002 & 2.103 & $15-59$ & $2 / 1$ & Mercúrio & 24,3 \\
\hline Bambuí & Barreto et al. 21 & 1997 & 2.314 & $\geq 18$ & $3 / 1$ & $\mathrm{NI}$ & $24,8 * \star$ \\
\hline Vitória & Mill et al. 23 & 2004 & 1.656 * & $25-64$ & $2 / 1$ & $\mathrm{NI}$ & 38,2 ** \\
\hline Salvador & Lessa et al. 39 & 2000 & 1.439 * & $\geq 20$ & $6 / 1$ & Eletrônico & 29,9 ** \\
\hline
\end{tabular}

NI: dados não informados.

* Amostra estratificada pelo nível sócio-econômico;

** HAS pelo critério de pressão arterial $\geq 140 \times 90 \mathrm{mmHg}$ mais uso de anti-hipertensivo;

*** População urbana e rural;

\# Pressão arterial corrigida pelo perímetro braquial. 
to, foi realizado o cálculo da prevalência ajustada para idade, cujo valor foi de $29,9 \%$.

Dentre outros fatores apontados na literatura para as discrepâncias nos valores de prevalência entre vários países 42 , em recente análise do estudo NHANES, foi encontrada correlação entre variações regionais de dieta e pressão arterial entre populações nos Estados Unidos 43. Em Tubarão, como há influência da imigração italiana e alemã, os hábitos alimentares talvez possam justificar em parte a alta prevalência de HAS, uma vez que a dieta nestes grupos está tradicionalmente associada a um consumo de sódio acima da média.

\section{Conhecimento, tratamento e controle da HAS}

O nível de conhecimento da HAS nos países economicamente desenvolvidos varia de $37 \%$ a $68,9 \% 5$

$\mathrm{O}$ percentual de indivíduos hipertensos em tratamento varia amplamente no mundo, desde valores razoáveis, como 58,4\% nos Estados Unidos, até valores muito baixos, como no México, onde apenas 10,7\% dos hipertensos estão sob tratamento 5 .

Com relação ao controle da hipertensão, o quadro é ainda mais alarmante, visto que nos Estados Unidos, país onde foi relatado o maior nível de controle da HAS, apenas 31\% dos hipertensos estão com cifras pressóricas abaixo de 140 x $90 \mathrm{mmHg} 43$.

No Brasil, poucos autores avaliaram dados sobre conhecimento, tratamento e controle de HAS em estudos de prevalência 11,12,16,17,18,23.

Em Porto Alegre, Estado do Rio Grande do Sul, Fuchs et al. 12 encontraram 42,3\% de hipertensos que sabiam de sua condição, 35,5\% dos quais estavam controlados. Em Catanduva, Estado de São Paulo 11, verificou-se haver $77 \%$ de conhecimento sobre serem hipertensos, dos quais $61,8 \%$ tratavam-se e apenas $17 \%$ estavam controlados. Em Campos dos Goytacazes, Estado do Rio de Janeiro 16, entre os pacientes hipertensos, 29,9\% sabiam da doença, 77,5\% faziam algum tipo de tratamento e $35,2 \%$ eram controlados. Gus et al. 17, no Rio Grande do Sul, revelaram que, entre os hipertensos, $50,8 \%$ conheciam sua condição, $40 \%$ tratavam e apenas $10,4 \%$ estavam em tratamento e controlados.

Em Tubarão, pouco mais da metade dos hipertensos conhecia sua condição $(44,4 \%$ eram hipertensos sem diagnóstico), e apesar de meta- de estar em tratamento, apenas 10,1\% estavam controlados, taxa semelhante à encontrada no Rio Grande do Sul 17.

Chama a atenção o VPP da entrevista, em relação ao questionamento sobre saber ser hipertenso, que foi superior ao VPN. Assim, a informação dada pelos indivíduos foi mais confiável em relação à condição de ser hipertenso do que à de não ser hipertenso. Daí a importância de que campanhas sejam feitas para o diagnóstico dessa condição, que cursa muitas vezes assintomática.

Observou-se neste estudo, assim como em estudos do Canadá 44, Estados Unidos e outros países 5 , além de em alguns estudos nacionais 19,22, que as mulheres têm maior conhecimento de sua condição de hipertensão, tratam e controlam mais do que os homens. Uma possível explicação para esse fato deve-se à já reconhecida maior preocupação da mulher com sua saúde, o que é confirmado pelo Hiperdia (Sistema de Cadastramento e Acompanhamento de Hipertensos e Diabéticos), programa do Ministério da Saúde destinado a cadastrar e acompanhar indivíduos hipertensos e diabéticos nos municípios do Brasil, que, em Tubarão, tem 4.559 hipertensos cadastrados, sendo $71 \%$ do sexo feminino 45 .

Chama a atenção neste estudo a alta prevalência de hipertensão na área urbana da cidade, o baixo grau de conhecimento de sua condição de hipertenso e a baixíssima taxa de controle, mesmo nos indivíduos em tratamento, apontando para a necessidade de que as ações públicas de saúde já existentes na cidade sejam implementadas. O fato de que as mulheres tiveram um nível maior de conhecimento, tratamento e controle da hipertensão em relação aos homens sugere a necessidade de que programas de detecção e acompanhamento desse agravo à saúde sejam dirigidos principalmente para os indivíduos do sexo masculino.

\section{Limitações do estudo}

A prevalência de HAS neste estudo pode ter sido superestimada, em virtude de as medidas da pressão arterial terem sido obtidas em uma única visita. Esse efeito pode ter sido minimizado pelo fato de terem sido desprezados os valores de pressão arterial com uma diferença maior que $6 \mathrm{mmHg}$ entre si, obtendo-se a média das duas últimas medidas mais próximas. 


\section{Resumo}

O objetivo deste estudo foi estimar a prevalência, o conhecimento, o tratamento e o controle de hipertensão arterial sistêmica (HAS) na população urbana adulta de Tubarão, Santa Catarina, Brasil. Foi realizado um estudo transversal, com uma amostra estratificada a partir de uma listagem dos pontos de luz, do qual participaram 707 indivíduos maiores de 18 anos. Foi aplicado um questionário, aferidas duas medidas da pressão arterial por manômetro de mercúrio, em uma visita domiciliar conduzida por estudantes de medicina. A prevalência de HAS pelo critério do The Seventh Report of The Joint National Committee on the Prevention, Detection, Evaluation, and Treatment of $\mathrm{Hi}-$ gh Blood Pressure (pressão arterial $\geq 140 \times 90 \mathrm{mmHg}$ ) foi de $36,4 \%$ e de 40,5\% quando acrescentados os indivíduos em uso de anti-hipertensivos. Tinham conhecimento da hipertensão 55,6\%; estavam em tratamento farmacológico 46,8\% e estavam com a pressão arterial controlada 10,1\% dos hipertensos. A elevada prevalência estimada de hipertensão arterial e os percentuais de conhecimento sobre o diagnóstico, tratamento e controle aquém do ideal apontam para necessidade de ações preventivas em Tubarão.

Hipertensão; Prevalência; Saúde do Adulto

\section{Colaboradores}

M. R. Pereira participou da concepção, planejamento, coordenação, coleta, análise, interpretação dos dados e elaboração do texto final. M. S. S. A. Coutinho colaborou no planejamento, análise, interpretação dos dados, revisão crítica do conteúdo e aprovação da versão final do manuscrito. P. F. Freitas contribuiu na análise e interpretação dos dados. E. D’Orsi participou da análise, interpretação dos dados e elaboração do texto final. A. Bernardi e R. Hass colaboraram na coleta dos dados e no planejamento.

\section{Agradecimentos}

Rafael Ronsoni, Ludovico S. Junior, Otávio Michels, Ivan C. Becker, Adriano Serafim, Ângela Dalmoro, Clarissa Bottura, Karina Silvestre, Thiago Dematê, Eduardo Antonelli, Lúcia Oliveira e Fabrício Foppa.

\section{Referências}

1. Burt VL, Cutler JA, Higgins M, Horan MJ, Labharthe D, Whelton P, et al. Trends in the prevalence, awareness, treatment, and control of hypertension in the adult US population: data from the health examination surveys, 1960 to 1991. Hypertension 1995; 26:60-9.

2. Levy D, Larson MG, Vasan RS, Kannel WB, Kalon KL. The progression from hypertension to congestive heart failure. JAMA 1996; 275:1557-62.

3. Chobanian AV, Bakris GL, Black HR, Cushman WC, Green LA, Izzo Jr. JL, et al. The Seventh Report of the Joint National Committee on Prevention, Detection, Evaluation, and Treatment of High Blood Pressure: the JNC 7 report. JAMA 2003; 289:2560-72.

4. Lloyd-Jones DM, Larson MG, Beiser A, Levy D. Lifetime risk of developing coronary heart disease. Lancet 1999; 353:89-92.

5. Kearney PM, Whelton M, Reynolds K, Whelton PK, He J. Worldwide prevalence of hypertension: a systematic review. J Hypertens 2004; 22:11-9.
6. Wang Y, Wang QJ. The prevalence of pre-hypertension and hypertension among US adults according to the New Joint National Committee Guidelines. Arch Intern Med 2004; 164:2126-34.

7. Joffres MR, Hamet P, MacLean DR, Litalien GJ, Fodor G. Distribution of blood pressure and hypertension in Canada and the United States. Am J Hypertens 2001; 14(11 Pt 1):1099-105.

8. Saldaña JR. Prevalencia de HAS. Arch Inst Cardiol Méx 1998; 68:131-9.

9. Fuchs SC, Petter JG, Accordi MC, Zen VL, Pizzol Jr. AD, Moreira LB, et al. Estabelecendo a prevalência de hipertensão arterial sistêmica: influência dos critérios de amostragem. Arq Bras Cardiol 2001; 76:445-8.

10. Gus I, Fischmann A, Medina C. Prevalência dos fatores de risco da doença arterial coronariana no Estado do Rio Grande do Sul. Arq Bras Cardiol 2002; 78:478-83. 
11. Freitas OC, Carvalho FR, Neves JM, Veludo PK, Parreira RS, Gonçalves RM, et al. Prevalência da hipertensão arterial sistêmica na população urbana de Catanduva. Arq Bras Cardiol 2001; 77:9-15.

12. Fuchs FD, Moreira LB, Moraes RS, Bredemeier M, Cardozo SC. Prevalência de hipertensão arterial sistêmica e fatores associados na região urbana de Porto Alegre: estudo de base populacional. Arq Bras Cardiol 1995; 63:473-9.

13. Lolio CM. Prevalência da hipertensão arterial em Araraquara. Arq Bras Cardiol 1990; 55:167-73.

14. Salas-Martins I, Marucci MFN, Velásquez-Meléndez G, Teixeira-Coelho L, Cervato AC. Doenças cardiovasculares ateroscleróticas, dislipemias, hipertensão, obesidade e diabetes melito em população da área metropolitana da região Sudeste do Brasil. III - Hipertensão. Rev Saúde Pública 1997; 31:466-71.

15. Ayres JEM. Prevalência da hipertensão arterial na cidade de Piracicaba. Arq Bras Cardiol 1991; 57:33-6.

16. Souza LJ, Chalita FEB, Reis AFF, Teixeira CL, Bastos DA, Souto Filho JTD, et al. Epidemiologia da hipertensão arterial sistêmica em Campos dos Goytacazes/RJ. In: Couto AA, Kaiser SE, organizadores. Manual de hipertensão arterial da Sociedade de Hipertensão do Estado do Rio de Janeiro (SOHERJ) Rio de Janeiro: Lemos Editorial; 2003. p. 26-35.

17. Gus I, Harzheim E, Zaslavsky C, Medina C, Gus M. Prevalência, reconhecimento e controle da hipertensão arterial sistêmica no Estado do Rio Grande do Sul. Arq Bras Cardiol 2004; 83:424-8.

18. Trindade IS, Heineck G, Machado JR, Ayzemberg H, Formighieri M, Crestani M, et al. Prevalência da hipertensão arterial sistêmica na população urbana de Passo Fundo. Arq Bras Cardiol 1998; 71:127-30.

19. Marcopito LF, Rodrigues SSF, Pacheco MA, Shirassu MM, Goldfeder AJ, Moraes MA. Prevalência de alguns fatores de risco para doenças crônicas na cidade de São Paulo. Rev Saúde Pública 2005; 39:738-45.

20. Klein CH, Silva NAS, Nogueira AR, Bloch KV, Campos LHS. Hipertensão arterial na Ilha do Governador, Rio de Janeiro, Brasil. I. Metodologia. Cad Saúde Pública 1995; 11:187-201.

21. Barreto SM, Passos VMA, Firmo JOA, Guerra HL, Vidigal PG, Lima-Costa MFF. Hypertension and clustering of cardiovascular risk factors in a community in Southeast Brazil - the Bambuí Health and Ageing Study. Arq Bras Cardiol 2001; 77:57681.

22. Firmo JOA, Uchôa E, Lima-Costa MF. Projeto Bambuí: fatores associados ao conhecimento da condição de hipertenso entre idosos. Cad Saúde Pública 2004; 20:512-21.

23. Mill JG, Ferreira AVL, Herkenhoff FL, Cunha RS, Molina MCB, Silva IO. Epidemiologia da hipertensão arterial na cidade de Vitória. Hipertensão 2004; 7:109-16.

24. Piccini RX, Victoria CG. Hipertensão arterial sistêmica em área urbana no Sul do Brasil: prevalência e fatores de risco. Rev Saúde Pública 1994; 28:2617.
25. Neder MM, Borges AAN. Hipertensão arterial sistêmica no Brasil: o que avançamos no conhecimento de sua epidemiologia. Rev Bras Hipertens 2006; 13:126-33.

26. Lessa I. Epidemiologia da hipertensão arterial e da insuficiência cardíaca no Brasil. Rev Bras Hipertens 2001; 8:383-92.

27. Departamento de Informática do SUS. Assistência à saúde: epidemiologia e morbidade. http://www. datasus.gov.br (acessado em 03/Set/2003).

28. Instituto Brasileiro de Geografia e Estatística. Censo demográfico 2000. Rio de Janeiro: Instituto Brasileiro de Geografia e Estatística; 2000.

29. Instituto Brasileiro de Geografia e Estatística. Censo demográfico 1991. Rio de Janeiro: Instituto Brasileiro de Geografia e Estatística; 1991.

30. Pereira MG. Epidemiologia teoria e prática: seleção dos participantes para estudo. Rio Janeiro: Editora Guanabara Koogan; 2000.

31. The sixth report of the Joint National Committee on Prevention, Detection, Evaluation, and Treatment of High Blood Pressure. Arch Intern Med 1997; 157:2413-46.

32. Sociedade Brasileira de Cardiologia. IV diretrizes brasileiras de hipertensão. Campos do Jordão: Sociedade Brasileira de Cardiologia; 2002.

33. Kirkwood BR. Essentials of medical statistics. New York: Blackwell Science; 2001.

34. Instituto Brasileiro de Geografia e Estatística. Censos demográficos e contagem populacional, para os anos intercensitários, estimativas preliminares dos totais populacionais, estratificada por idade e sexo. http://www.saude.sc.gov.br (acessado em 04/Jan/2005).

35. Colton T. Statistics in medicine. London: Little Brown and Co.; 1974.

36. Duda NT, Lisboa HRK, Portella M, Krahl M, Portela SN, Giengo BM. Hipertensão arterial sistêmica: epidemiologia e prevenção no Rio Grande do Sul. Arq Bras Cardiol 1994; 63:445-9.

37. Cavalcante JWS, Daza CMG, Cavalcante LP, Pacheco WS, Menezes MGF, Melo R, et al. Prevalência e aspectos sócio-culturais e econômicos da hipertensão arterial em centro de saúde da zona norte de Manaus. Arq Bras Cardiol 1995; 65:493-6.

38. Birkett NJ. The effect of alternative criteria for hypertension on estimates of prevalence and control. J Hypertens 1997; 15:237-44.

39. Lessa I, Magalhães L, Araújo MJ, Almeida Filho N, Aquino E, Oliveira MMC. Hipertensão arterial na população adulta de Salvador (BA) - Brasil. Arq Bras Cardiol 2006; 87:747-56.

40. Dressler WW, Santos JE. Social and cultural dimensions of hypertension in Brazil: a review. Cad Saúde Pública 2000; 16:303-15.

41. Molina MCB, Cunha RS, Herckenhoff LF, Mill JG. Hipertensão arterial e consumo de sal em população urbana. Rev Saúde Pública 2003; 37:743-50.

42. Wolf-Maier K, Cooper RS, Banegas JR, Giampaoli S, Hense HW, Joffres M, et al. Hypertension prevalence and blood pressure levels in 6 European Countries, Canada, and the United States. JAMA 2003; 289:2363-9 
43. Hajjar I, Kotchen TA. Trends in prevalence, awareness, treatment and control of hypertension in the United States, 1988-2000. JAMA 2003; 290:199-206.

44. Joffres MR, Ghadirian P, Fodor JG, Petrasovits A, Chockalingam A, Hamet P. Awareness, treatment, and control of hypertension in Canada. Am J Hypertens 1997; 10(10 Pt 1):1097-102.
45. Ministério da Saúde. Relatórios. http://hiperdia. datasus.gov.br (acessado em 19/Fev/2007).

46. Instituto Brasileiro de Geografia e Estatística. Censo demográfico 2003. Rio de Janeiro: Instituto Brasileiro de Geografia e Estatística; 2003.

Recebido em 03/Ago/2006

Versão final reapresentada em 15/Mar/2007

Aprovado em 16/Abr/2007 